\title{
Mapping Social Dynamics on Facebook: The Brexit Debate
}

\author{
Michela Del Vicario ${ }^{1, *}$ \\ CSSLab, IMT School for Advanced Studies, Lucca, Italy \\ Fabiana Zollo ${ }^{1, *}$ \\ Dept. of Environmental Sciences, Informatics and Statistics \\ Ca' Foscari University of Venice, Venice, Italy \\ Antonio Scala* \\ ICS CNR, Rome, Italy \\ Guido Caldarelli* \\ CSSLab, IMT School for Advanced Studies, Lucca, Italy \\ Walter Quattrociocchi* \\ CSSLab, IMT IMT School for Advanced Studies, Lucca, Italy
}

\begin{abstract}
Nowadays users get informed and shape their opinion through social media. However, the disintermediated access to contents does not guarantee quality of information. Selective exposure and confirmation bias, indeed, have been shown to play a pivotal role in content consumption and information spreading. Users tend to select information adhering (and reinforcing) their worldview and to ignore dissenting information. This pattern elicits the formation of polarized groups - i.e., echo chambers - where the interaction with like-minded people might even reinforce polarization. In this work we address news consumption around Brexit in UK on Facebook. In particular, we perform a massive analysis on more than 1 Million users interacting with Brexit related posts from
\end{abstract}

\footnotetext{
* Corresponding author

Email address: michela.delvicario@imtlucca.it (Michela Del Vicario)

${ }^{1}$ These authors contributed equally to this work.
} 
the main news providers between January and July 2016. We show that consumption patterns elicit the emergence of two distinct communities of news outlets. Furthermore, to better characterize inner group dynamics, we introduce a new technique which combines automatic topic extraction and sentiment analysis. We compare how the same topics are presented on posts and the related emotional response on comments finding significant differences in both echo chambers and that polarization influences the perception of topics. Our results provide important insights about the determinants of polarization and evolution of core narratives on online debating.

Keywords: Collective Debates, Polarization, Online Social Networks

\section{Introduction}

The Arab Spring and Ukrainian revolution showed social media as a liberalizing technology and powerful vehicle of information, engagement, mobilization, able to encourage innovation and democracy. But social media have also changed the way we get informed and form our opinions.

According to a recent report [1, approximately $63 \%$ of users acquire their news from social media, and these news stories undergo the same popularity dynamics as other forms of online contents (such as selfies and cat photos). As a result of disintermediated access to information and of algorithms used in content promotion, communication has become increasingly personalized, both in the way messages are framed and how they are shared across social networks.

Selective exposure and confirmation bias, indeed, have been shown to play a pivotal role in content consumption and information spreading [2]. Users tend to select information adhering (and reinforcing) their worldview and to ignore dissenting information [3, 4, 5, 6, This pattern elicits the formation of polarized groups - i.e., echo chamber - where the interaction within like-minded people might even reinforce polarization [7, 8].

Several studies pointed out the effects of social influence online [9, 10, 11, 12, Results reported in 13 indicate that emotions expressed by others on Facebook 
influence our own emotions, providing experimental evidence of massive-scale contagion via social networks. Recent works [6, 5, indeed, showed that attempts to debunk false information are largely ineffective. In particular, the discussion degenerates when the two polarized communities interact with one another. The increasing interest in online debates led researchers to investigate many 25 of their aspects, from the characterization of conversation threads [14 to the detection of bursty topics on microblogging platforms [15], to the disclosure of the mechanisms behind information diffusion for different kinds of contents [16].

More recently, several doubts about social influence on the Internet have been raised during Brexit - the British referendum to leave the European Unioncampaign, where both sides, Leave and Remain, battled it out on social media. Indeed, a big effort has been dedicated to characterize the dynamics of the online Brexit debate, from applying filtering algorithms to study the shape of online data [17, through the investigation of the role of bots on the direction of discussions [18, to the study of the effects of the referendum result on financial 35 markets [19].

In this paper we address the Brexit discussion on Facebook public pages referring to UK based official information sources listed in the European Media Monitor [20].

Firstly, we characterize the structural properties of the discussion by ob40 serving the spontaneous emergence of two well-separated communities; indeed, connections among pages are the direct result of users' activity, and we do not perform any categorization of contents a priori. Then, we explore the dynamics behind discussion: looking at users polarization towards the two communities and at their attention patterns, we find a sharply bimodal distribution, showing that users are divided into two main distinct groups and confine their attention on specific pages.

Finally, to better characterize inner group dynamics, we introduce a new technique which combines automatic topic extraction and sentiment analysis. We compare how the same topics are presented on posts and the related com50 ments, finding significant differences in both echo chamber and that polarization 
on the perception of topics. We first measure the distance between how a certain concept is presented on the posts and then the emotional response of users to such controversial topics. Our new metrics could be of great interest to identify the most crucial topics in online debates. Indeed, it is highly likely that the greater the emotional distance between the same concept in two echo chambers, the greater the polarization of users involved in the discussion. Therefore, such a distance may be a key marker to locate controversial topics and to understand the evolution of the core narratives within distinct echo chambers.

\section{Methods}

\section{Ethics Statement.}

The data collection process was carried out using the Facebook Graph API [21], which is publicly available. For the analysis (according to the specification settings of the API) we only used publicly available data (thus users with privacy restrictions are not included in the dataset). The pages from which we downloaded data are public Facebook entities and can be accessed by anyone. Users' content contributing to such pages is also public unless users' privacy settings specify otherwise, and in that case it is not available to us.

\section{Data collection.}

The European Media Monitor (EMM) 20] provides a list of all news sources which includes, for each of them, the related country and region. We limited our collection to all pages whose legal head office (at least one of them) is located in the United Kingdom. For each page, we downloaded all the posts from January 1st to July 15th, 2016, as well as all the related likes and comments. The exact breakdown of data is provided in Tab. 2, while the complete set of downloaded 75 pages is reported in Tab. 5 in Appendix B.

Preliminaries and Definitions.

In this section we provide a brief description of the main concepts and tools used in the analysis. 


\begin{tabular}{|c|c|c|}
\hline & Total & Brexit \\
\hline \hline Pages & 81 & 38 \\
Posts & 303,428 & 5,039 \\
Likes & $186,947,027$ & $2,504,956$ \\
Comments & $38,182,541$ & 469,397 \\
Likers & $30,932,388$ & $1,365,821$ \\
Commenters & $7,222,273$ & 259,078 \\
\hline
\end{tabular}

Table 1: Dataset description: from January 1st to July 15th, 2016.

\section{Bipartite Projection.}

A bipartite graph is a triple $\mathcal{G}=(A, B, E)$ where $A=\left\{a_{i} \mid i=1 \ldots n_{A}\right\}$ and $B=\left\{b_{j} \mid j=1 \ldots n_{B}\right\}$ are two disjoint sets of vertices, and $E \subseteq A \times B$ is the set of edges - i.e. edges exist only between vertices of the two different sets $A$ and $B$. The bipartite graph $\mathcal{G}$ is described by the rectangular matrix $M$ defined as

$$
M_{i j}=\left\{\begin{array}{cc}
1 & \text { if an edge exists between } a_{i} \text { and } b_{j} \\
0 & \text { otherwise }
\end{array} .\right.
$$

We consider the bipartite network $\mathcal{G}=(P, U, E)$ where $P$ is the set of Facebook pages concerned on Brexit topics (see Tab. 5 in Appendix B) and $U$ is the set of users active on pages belonging to $P$. An interaction with a given information posted by a page $p \in P$ determines a link between a user $u \in U$ and the page $p$, hence $M_{p, u}=1$ indicates that user $u$ was active on page $p$. For our analysis we use the co-occurrence matrices $C^{P}=M M^{T}$ and $C^{U}=M^{T} M$ that count, respectively, the number of common neighbors between two vertices of $P$ or $U$. As an example, $C_{p, q}^{P}$ for $p \neq q$ counts the number of users that were active on both pages $p$ and $q . C^{P}$ can be interpreted as the weighted adjacency matrix of the co-occurrence graph $G^{P}$ with vertices on $P$. Each non-zero element $C_{p, q}^{P}$ 95 corresponds to an edge $(p, q)$ among vertices $p$ and $q$ with weight $C_{p q}^{P}$. 


\section{Community Detection Algorithms.}

Community detection algorithms serve to identify groups of nodes in a network. Most of the strategies relies on the modularity which quantifies the division of a network in separated clusters, high modularity corresponds to a dense connectivity between nodes in a community and sparse connections between modules. In this work we apply four different community detection algorithms: Fast Greedy (FG), that seeks for the maximum modularity score by considering all possible community structures in the network. It tries to optimize the modularity function in a greedy manner [22. Walktrap (WT), that exploits the fact that a random walker tends to remain trapped in the denser part i.e., communities - of a graph. Hence WT uses short random walks to merge separate communities [23. Multilevel (ML), that is based on a multi-level modularity optimization procedure [24]. Label Propagation (LP) 25], that is a nearly linear time algorithm that gives unique labels to vertices that are then updated according to majority voting in the neighboring vertices. Dense group of nodes reach consensus on a common label quickly. To compare the various community structures we use standard methods that compute the similarity between different clustering methods by considering how nodes are assigned by each community detection algorithm [26, 27.

\section{Backbone Detection Algorithm.}

The disparity filter algorithm is a network reduction technique based on the local identification of the statistically relevant weight heterogeneities. This method is able to identify the backbone structure of a weighted network without destroying its multi-scale nature 28. We make use of this algorithm to obtain the relevant connections that form our networks' backbones and produce clearer visualizations.

\section{Results and Discussion}

As a preliminary step, we divide all UK based pages in two groups: Brexit pages, that includes those pages engaged in the debate around the Brexit, and 
the Brexit. Hence, we characterize the users behavior on Brexit pages and their related posts.

\subsection{Communities and News Polarization}

Online social media proved to facilitate the aggregation of individuals in communities of interest, also known as echo chambers [3], especially when restricting the interaction of users to conflicting information [2, 5]. In the case of Brexit pages we focus of the emerging communities without considering the shared contents, but rather by accounting for the connections created by users activities.

135 graph. We consider the bipartite projection of the pages-users graph $G_{p}$ where nodes are Brexit pages and two pages are connected if at least one user liked a post from each of them. The weight of a link is determined by the number of users in common between the two pages.

In Fig. 11(a) we show the backbone structure of $G_{p}$. Colors (resp., blue and red) represent the membership to one of the two communities (resp., C1 and C2) detected by the Fast Greedy (FG) algorithm (see Methods section for further details). Fig. 1(b) reports the percentage of pages in both communities. A complete list of the pages and the relative membership is reported in Tab. 5 in Appendix B. We compare the results of FG and two other community detection algorithms i.e., Walktrap (WT) and Multilevel (ML) (refer to Methods section for further details) by means of the Rand method [26, 27]; we find a very high concordance between FG and ML (0.90), and lower ones between FG and WT (0.69), and between ML and WT (0.63) ${ }^{2}$ Our analysis underlines the spontaneous emergence of two separate communities active on Brexit pages, where the connections among pages are a simple result of the interaction of users on them.

\footnotetext{
${ }^{2}$ For further details on the comparison between the community detection algorithms, and for a complete analysis of the WT case, refer to Appendix A.
} 


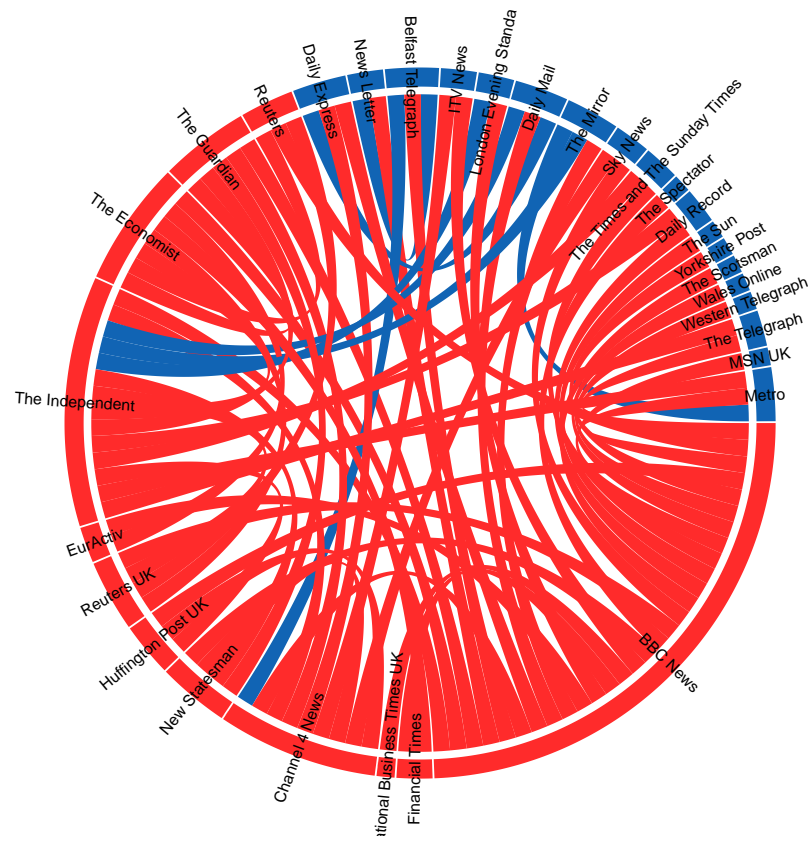

(a)

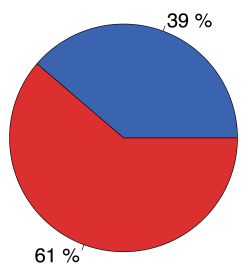

(b)

Figure 1: Backbone structure for the bipartite projection of the pages-users graph $G_{p}$ (a) and percentage of pages in the different communities (b). Colors indicate the membership of users in the different communities (blue for $\mathrm{C} 1$, red for $\mathrm{C} 2$ ) detected by the $\mathrm{FG}$ algorithm, while for the extraction of the backbone we considered the level of significance $\alpha=0.03$.

Taking into account the positive meaning of the like as a feedback to a post, we characterize how contents from the two communities detected in $G_{p}$ are consumed by Facebook users. We define the users polarization by likes (reps., comments) as

$$
\varrho(u)=(y-x) /(y+x),
$$

where $y$ is the number of likes (resp., comments) that user $u$ left on posts of $\mathrm{C} 1$ and $x$ the number of likes (resp., comments) left on posts of C2. Thus, a user $u$ is said to be polarized towards C1 (resp., C2) if $\varrho(u)=1$ (resp., -1). In Fig. 2 we report the Probability Density Function (PDF) of users polarization by likes (left panel) and comments (right panel). We find that $\varrho(u)$ is sharply bimodal in both cases, denoting that the majority of users may be divided into two main 


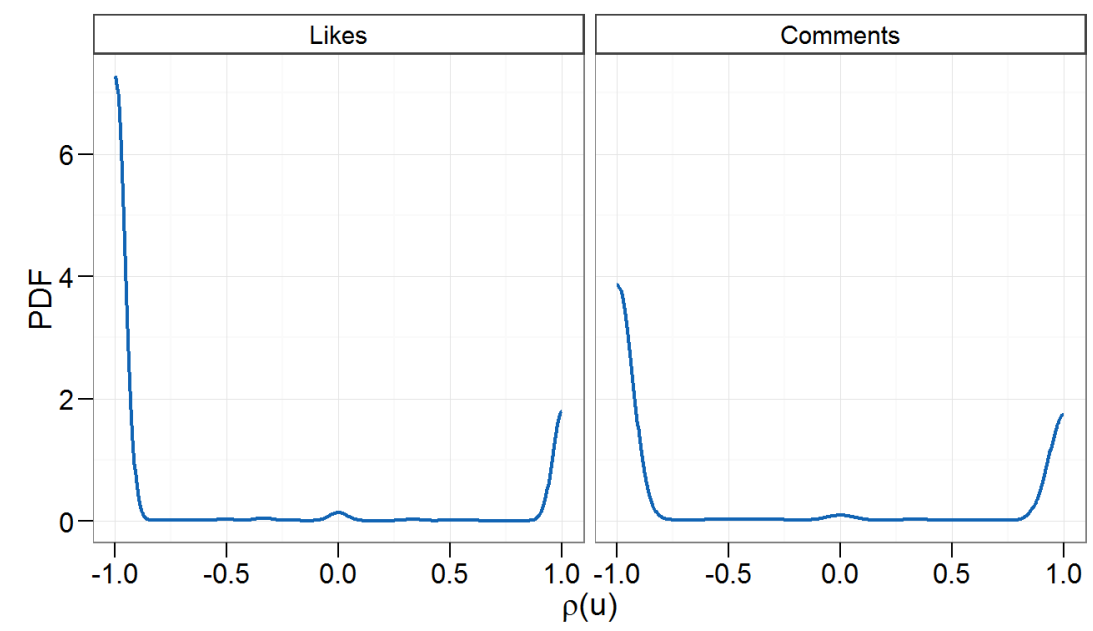

Figure 2: Probability density function (PDF) for the users polarization $\varrho(u)$ by likes (left) and by comments (right). $\varrho(u)=1$ (resp. $\varrho(u)=-1$ ) indicates that users $u$ is polarized towards C2 (resp., C1).

groups referring to the two communities of Fig. 11(a). Tab. 2 shows the number of polarized users towards both communities by likes and comments.

\begin{tabular}{|l|c|c|}
\hline & C1 & C2 \\
\hline \hline Likes & $1,037,969$ & 255,930 \\
Comments & 168,680 & 75,851 \\
\hline
\end{tabular}

Table 2: Number of polarized users towards both communities by likes and comments.

160 the Brexit debate shows features very similar to those already observed for other phenomena, such as political bloggers [29] or misinformation. In the latter case, researchers confirmed the existence of echo chambers both on Facebook [5, 6] and YouTube [30] and showed that users interact primarily with either conspiracylike or scientific pages. However, such a simmetry takes on a particular relevance in this work, because the emergence of the two echo chambers is completely spontaneous and no a priori categorization has been performed. 
We now want to compare users' activities on posts from Brexit pages. In Fig. 3(a) we report the Complementary Cumulative Distribution Function (CCDF) of likes (left) and comments (right) made by users polarized towards both communities, while in Fig. 3(b) we report the CCDF of the lifetime of polarized users. The lifetime is defined as the temporal distance, in terms of days, between the first and last comment made by any given user. We fitted the distributions in Fig. 3(a) with different models (the exponential, the power law, and the log-normal) by means of NLS estimation, goodness of fit tests are based on the maximization of the log-likelihood. Then, we pairwise compare the distributions of the number of likes and comments by users polarized towards either community by means of the Kolmogorov-Smirnov (KS) test. Results for the best fit and KS test are reported in Tab. 3. We may notice that the distributions are all best fitted by the exponential model, with the exception of that of the number of likes by users from $\mathrm{C} 2$, that is best fitted by the power-law. Also, we fail to reject the null hypothesis of equivalence of the two distributions in the case of the number of comments by users from either community.

\begin{tabular}{|c|c|c|}
\hline Distribution & Best Fit & Estimated Parameters \\
\hline \hline \# of likes (C1) & exponential & $\hat{a}=1.49, \hat{b}=0.00003$ \\
\# of likes (C2) & power-law & $\hat{a}=1.18, \hat{b}=-0.03$ \\
\# of comments (C1) & exponential & $\hat{a}=1.55, \hat{b}=0.00005$ \\
\# of comments (C2) & exponential & $\hat{a}=1.58, \hat{b}=0.0001$ \\
\hline
\end{tabular}

\begin{tabular}{|c|c|c|}
\hline \multicolumn{3}{|c|}{ KS test } \\
\hline \hline Compared Distributions & D (C) & p-value \\
\hline \hline \# of likes (C1/C2) & $0.075(0.004)$ & $2 \times 10^{-} 16$ \\
\# of comments (C1/C2) & $0.004(0.007)$ & 0.349 \\
\hline
\end{tabular}

Table 3: Fit of distributions from Fig. 3 and results of Kolmogorov-Smirnov tests.

Interestingly, although users tend to focus on contents coming just from one of the two communities, the distributions of their attention patterns are very 
similar, and even equal in the case of comments.

\subsection{Emotional Dynamics Inside and Between Communities}

Our analysis provides evidence of the existence of two well segregated echo chambers: users tend to focus on one narrative and to ignore the other. Such a pattern might be driven by the way in which contents are debated on pages, i.e., is such a way that matches their own users' preferences. To shade light on this aspect, we want to measure the distance among the sentiment of the users w.r.t. the same topic. Thus, we analyze how the subject of a post is presented to the users. To perform the analysis we make use of IBM Watson ${ }^{\text {TM }}$ AlchemyLanguage service API [31, that allows us to extract semantic meta-data from posts content. Such a procedure applies machine learning and natural language processing techniques aimed to analyze text by automatically extracting relevant entities, their semantic relationship as well as the emotional sentiment they express 32 . In particular, we extract the sentiment and the main concepts presented by each post of the dataset, whether it has a textual description or a link to an external document. The AlchemyAPI tools make use of the language patterns surrounding the input text looking for signals that denote the sentiment and exploring information based on the concepts behind such an input. Thus, a concept is a high-level conceptual association identified in the content provided as input to the service. Input content is auto-tagged against a concept graph, which formally represents the relationships between the concepts contained in the data on which it is based.

Fig. 4 shows the sentiment distribution of posts on both communities. The sentiment score is defined in the range $[-1,1]$, where -1 is negative, 0 is neutral, and 1 is positive. We may observe a negative overall pattern for both categories, although clearly more pronounced for posts of $\mathrm{C} 1$. Notice that we consider how subjects are presented in a post; here we do not take into account the sentiment that the post may elicit in the reader, or the sentiment of users involved in the discussion. 


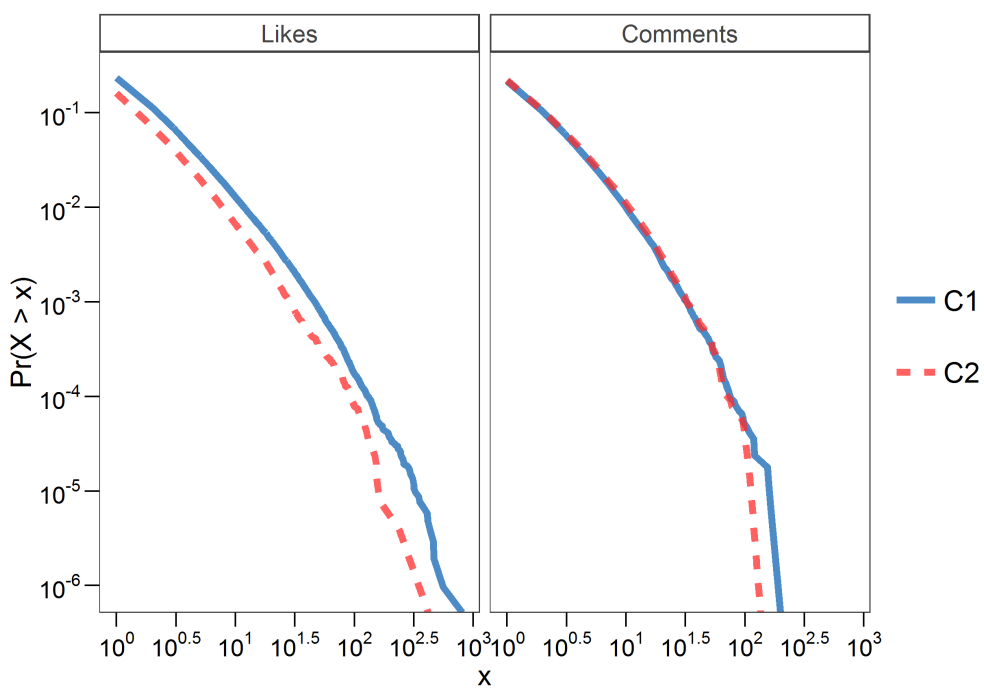

(a)

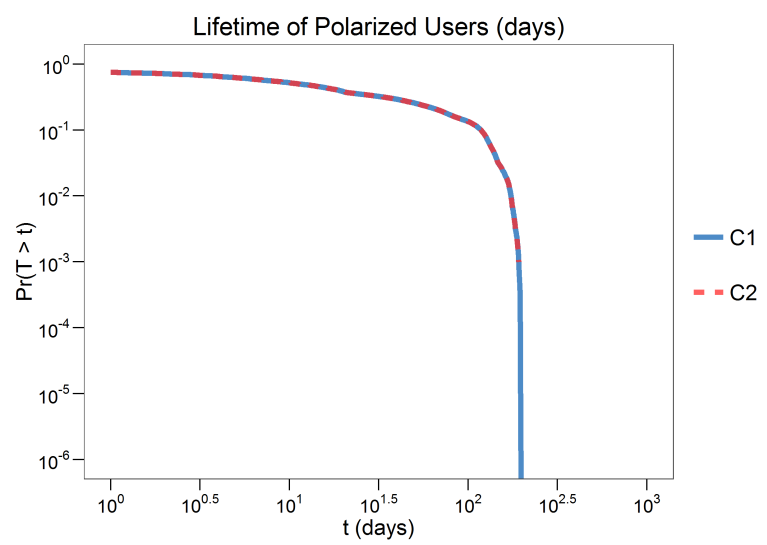

(b)

Figure 3: (a) CCDF of likes (left) and comments (right) made by users polarized towards C1 (solid blue) and towards $\mathrm{C} 2$ (dashed red). (b) CCDF of the lifetime of users polarized towards either $\mathrm{C} 1$ (solid blue) or 2 (dashed red). The lifetime is computed as the temporal distance, in terms of days, between the first and last comment made by any given user. 


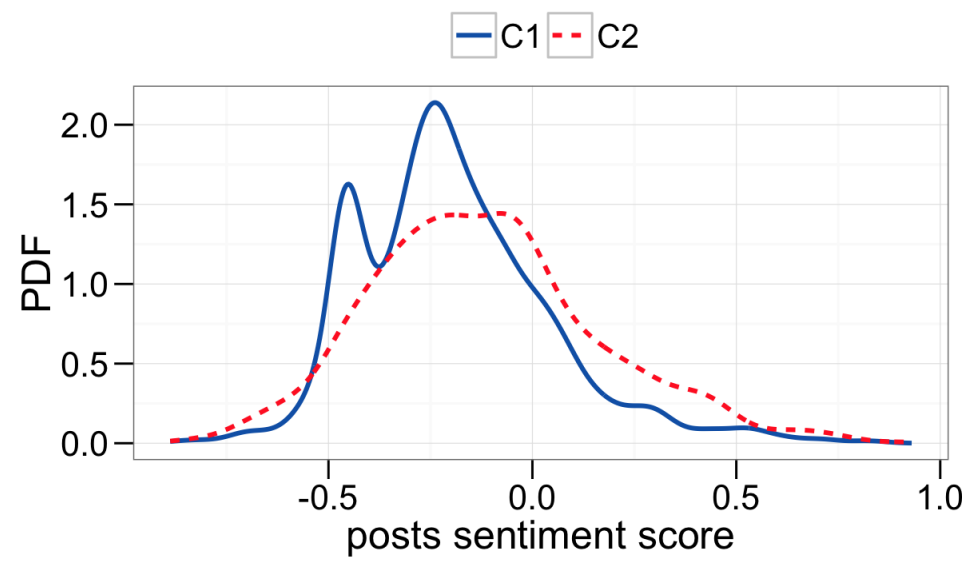

Figure 4: Probability Density Function (PDF) of posts sentiment score on C1 (solid blue) and $\mathrm{C} 2$ (dashed red). The sentiment score is defined in the range $[-1,1]$, where -1 is negative, 0 is neutral, and 1 is positive

Controversial Concepts: Emotional Distance and Users' Response

We now want to understand how users of the two echo chambers perceive the issues debated on their pages. Thus, we focus on the concepts of each echo chamber: 102 such concepts are shared by both communities, for a total of 1,520 posts $(1,258 \in C 1,262 \in C 2)$ and 115,958 comments $(95,357 \in C 1$, $20,601 \in C 2)$. For each concept we compute its average sentiment - i.e., the mean of the sentiment of all the posts where it appears. The emotional distance between two concepts is defined as the difference between the average sentiment of the concept in $C 2$ and that in $C 1$. Since we are interested in identifying the most controversial concepts, we consider only those concepts for which the emotional distance (in absolute value) between the two communities is greater than 0.2. Fig. 5 shows, for each concept, the emotional distance between the two echo chambers. More specifically, the top panel (a) of Fig. 5 includes the 52 concepts that are presented in a more negative way in community $\mathrm{C} 1$ w.r.t. $\mathrm{C} 2$, while the bottom panel (b) includes the 48 concepts that are presented in 
shown in descending order by the largest to the smallest emotional distance. Thus, concepts on the left are those discussed with the greatest difference in sentiment, while those on the right are discussed in a much more similar way by both echo chambers.

${ }_{235}$ So far we have analyzed how subjects are debated in the posts of Brexit pages. What about the emotional response of users to such posts? To this aim we take all the comments $(115,958)$ of posts including one of the concepts and compute their sentiment score through AlchemyAPI. Thus, to each comment is associated a sentiment score in $[-1,1]$, where -1 is negative, 0 is neutral, and 1 is positive. For each post (resp., user) we compute the average sentiment of its (resp., her) comments - i.e., the mean of the sentiment of all comments on the post (resp., made by the user). Then, for each concept, we consider the emotional distance between the average sentiment of the post and that of its users. Fig. 6 shows the emotional response of users to posts of $\mathrm{C} 1$ (a) and C2 (b) debating one of the listed controversial topics. Only concepts for which the emotional distance (in absolute value) between the two communities is greater than 0.2 have been taken into account. In both panels a vertical dashed line denotes a change in users' response: concepts on the left are those for which users' response is more negative than the sentiment expressed in the

250 post, and vice versa for those on the right. We may notice that users tend to react negatively to the content of the posts, independently of their reference community.

\section{Conclusions}

We address the online discussion around Brexit on Facebook by means of 255 a quantitative analysis on a sample of $5 \mathrm{~K}$ posts from 38 pages linked to official UK news sources. We observe the spontaneous emergence of two separate communities, where the connections among pages are the direct result of users' activity and no reference to the shared contents is implied. We further explore the dynamics of the discussion by looking at the polarization of users from the 


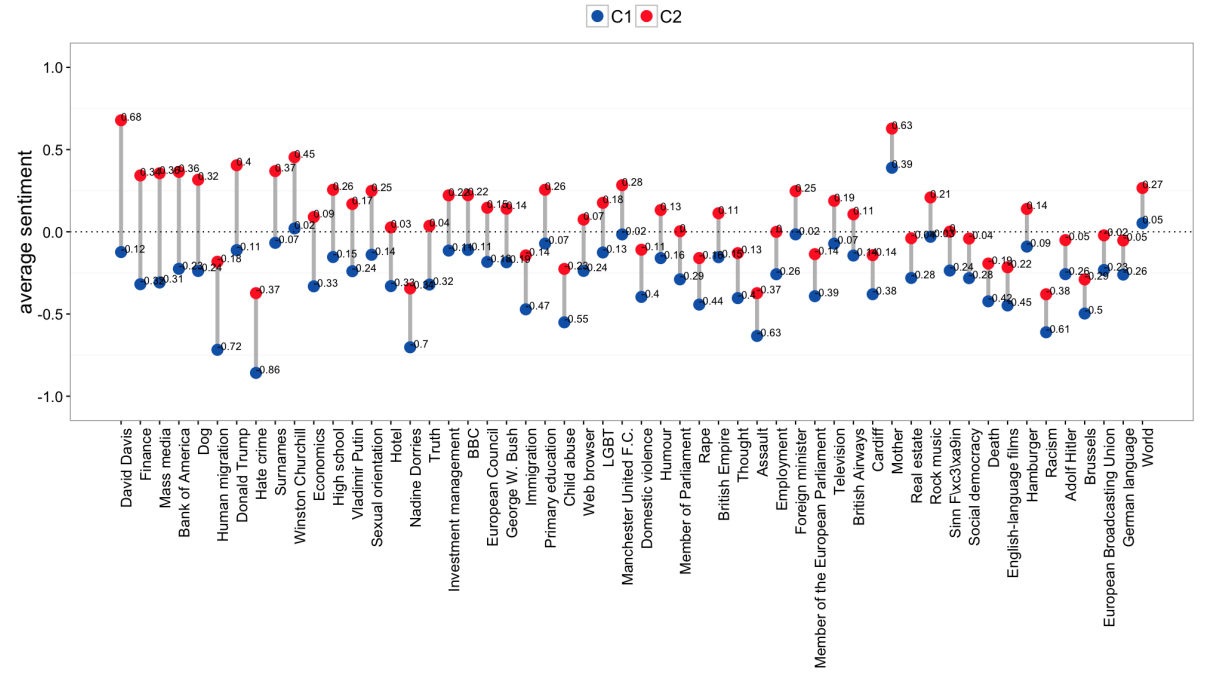

(a)

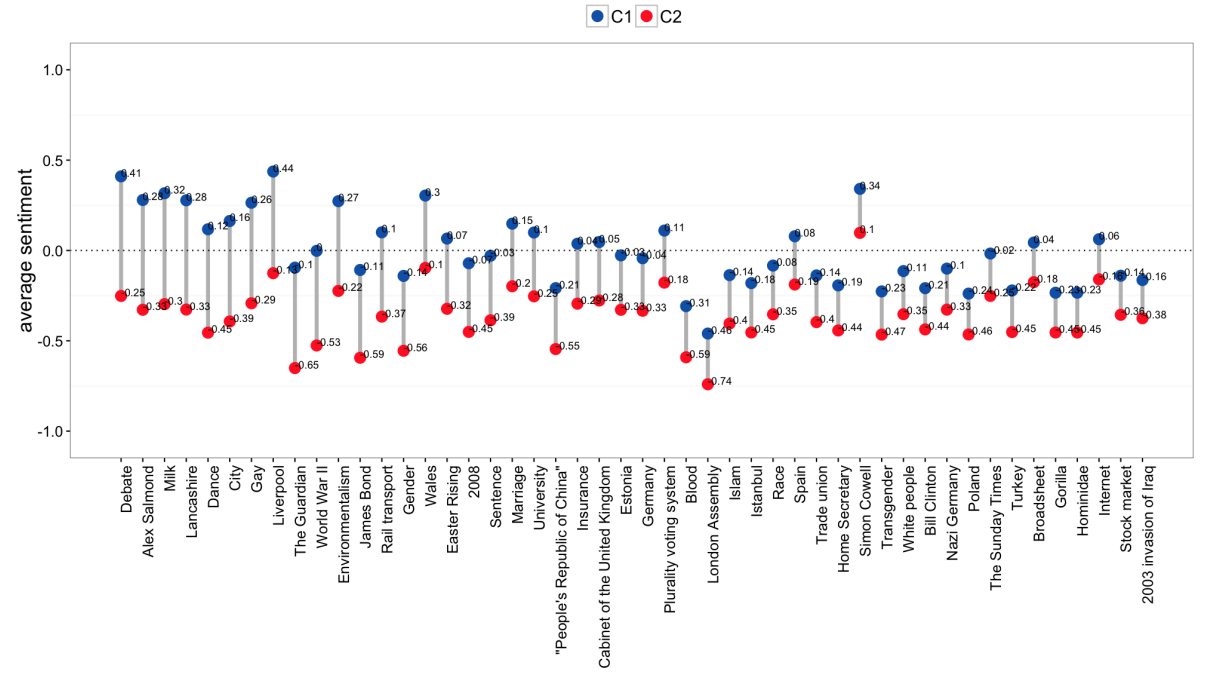

(b)

Figure 5: Emotional Distance Between Communities. Emotional distance - i.e., the distance between the average sentiment of a concept on both communities - for each concept debated in both communities. Panel (a) includes the 52 concepts that are presented in a more negative way in community C1 (blue dots) w.r.t. C2 (red dots), while panel (b) includes the 48 concepts that are presented in a more negative way in community $\mathrm{C} 2$ (red dots) w.r.t. C1 (blue dots). Concepts are shown in a descending order by the largest to the smallest emotional distance. 


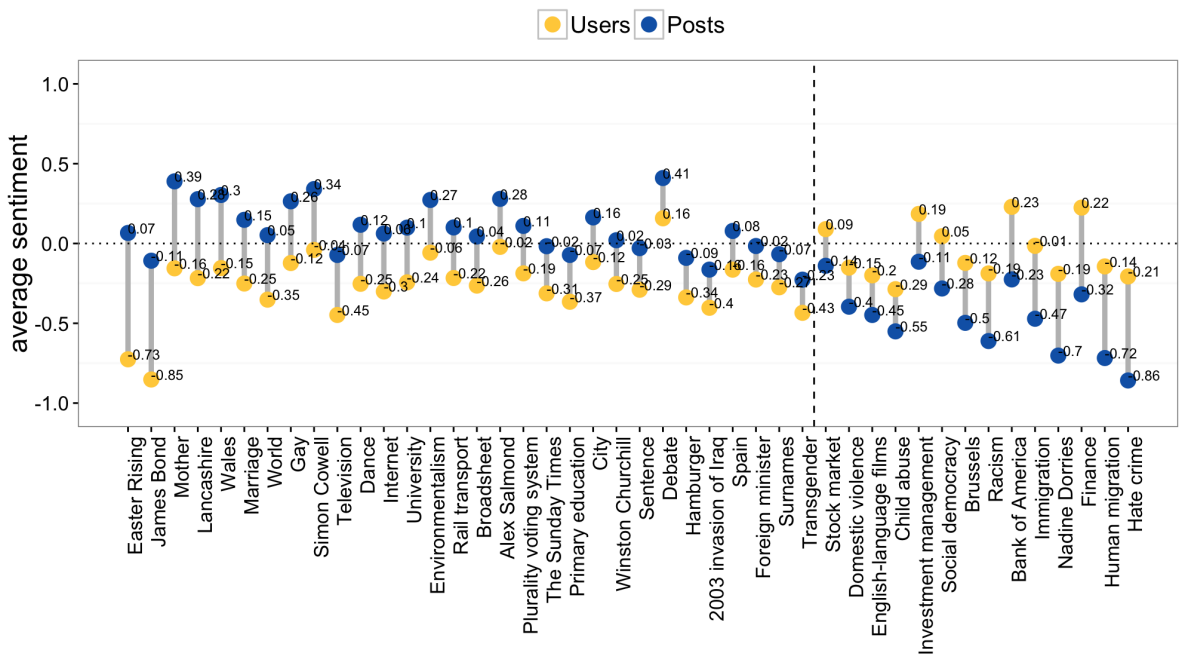

(a)

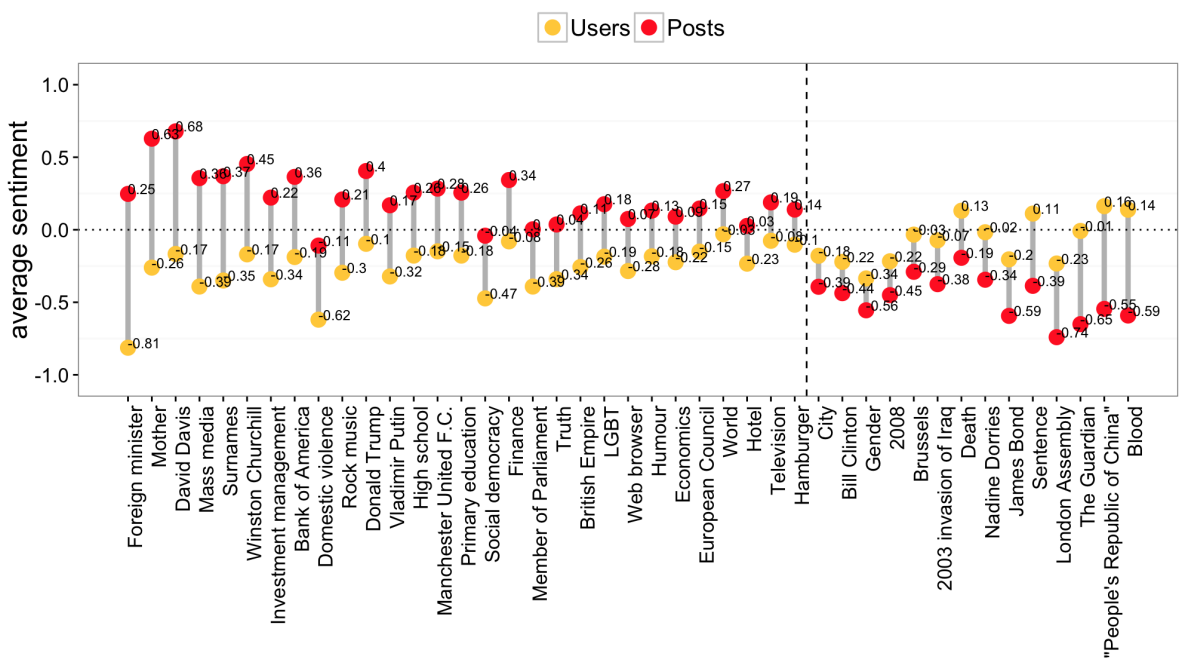

(b)

Figure 6: Emotional Response to Controversial Concepts. Panel (a) shows the emotional response of users (yellow dots) to posts of $\mathrm{C} 1$ (blue dots) debating one of the listed controversial concepts, while panel (b) shows the emotional response of users (yellow dots) to posts of $\mathrm{C} 2$ (red dots). Only concepts for which the emotional distance between the two communities is greater than 0.2 are reported. The vertical dashed lines denote a change in users' response. 
tribution for the polarization of users. Users segregation might be driven by the match between their personal preferences and the way in which contents are presented. We identify how concepts get received and shape the narrative inside the echo chamber by measuring both the distance between the sentiment topic. Firstly, we characterize the structural properties of the discussion by observing the spontaneous emergence of two well-separated communities; indeed, connections among pages are the direct result of users' activity, and we do not perform any categorization of contents a priori. Then, we explore the dynamics and at their attention patterns, we find a sharply bimodal distribution, showing that users are divided into two main distinct groups and confine their attention on specific pages. Finally, to better characterize inner group dynamics, we introduce a new technique which combines automatic topic extraction and and the related comments, finding significant differences in both echo chambers and that polarization reflects on the perception of topics. We first measure the distance between how a certain concept is presented on the posts by both echo chambers and then we measure the emotional response of users to such controversial topics. Our new measures could be of great interest to identify the most crucial topics in online debates. Indeed, it is highly likely that the greater the emotional distance between the same concept in two echo chambers, the greater users' polarization. Our results provide important insights for identifying the determinants of polarization and evolution of the core narratives behind online debating.

\section{References}

[1] N. Newman, D. A. Levy, R. K. Nielsen, Reuters institute digital news report 2015, Available at SSRN 2619576. 
[2] M. Del Vicario, A. Bessi, F. Zollo, F. Petroni, A. Scala, G. Caldarelli, H. E. Stanley, W. Quattrociocchi, The spreading of misinformation online, Proceedings of the National Academy of Sciences 113 (3) (2016) 554-559.

[3] W. Quattrociocchi, A. Scala, C. R. Sunstein, Echo chambers on facebook, Available at SSRN.

[4] A. Bessi, F. Petroni, M. Del Vicario, F. Zollo, A. Anagnostopoulos, A. Scala, G. Caldarelli, W. Quattrociocchi, Viral misinformation: The role of homophily and polarization, in: Proceedings of the 24th International Conference on World Wide Web Companion, International World Wide Web Conferences Steering Committee, 2015, pp. 355-356.

[5] F. Zollo, A. Bessi, M. Del Vicario, A. Scala, G. Caldarelli, L. Shekhtman, S. Havlin, W. Quattrociocchi, Debunking in a world of tribes, arXiv preprint arXiv:1510.04267.

[6] A. Bessi, M. Coletto, G. A. Davidescu, A. Scala, G. Caldarelli, W. Quattrociocchi, Science vs conspiracy: collective narratives in the age of misinformation, PloS one 10 (2) (2015) 02.

[7] F. Zollo, P. K. Novak, M. Del Vicario, A. Bessi, I. Mozetič, A. Scala, G. Caldarelli, W. Quattrociocchi, Emotional dynamics in the age of misinformation, PloS one 10 (9) (2015) e0138740.

[8] C. R. Sunstein, The law of group polarization, Journal of political philosophy 10 (2) (2002) 175-195.

[9] D. Centola, The spread of behavior in an online social network experiment, Science 329 (5996) (2010) 1194-1197.

[10] J. H. Fowler, N. A. Christakis, Cooperative behavior cascades in human social networks, Proceedings of the National Academy of Sciences 107 (12) (2010) 5334-5338. 
[11] W. Quattrociocchi, G. Caldarelli, A. Scala, Opinion dynamics on interacting networks: media competition and social influence, Scientific reports 4.

[12] M. J. Salganik, P. S. Dodds, D. J. Watts, Experimental study of inequality and unpredictability in an artificial cultural market, Science 311 (5762)

[13] A. D. I. Kramer, J. E. Guillory, J. T. Hancock, Experimental evidence of massive-scale emotional contagion through social networks, Proceedings of the National Academy of Sciences (2014) 8788-8790.

[14] L. Backstrom, J. Kleinberg, L. Lee, C. Danescu-Niculescu-Mizil, Characterizing and curating conversation threads: expansion, focus, volume, re-entry, in: Proceedings of the sixth ACM international conference on Web search and data mining, ACM, 2013, pp. 13-22.

[15] Q. Diao, J. Jiang, F. Zhu, E.-P. Lim, Finding bursty topics from microblogs, in: Proceedings of the 50th Annual Meeting of the Association for Computational Linguistics: Long Papers-Volume 1, Association for Computational Linguistics, 2012, pp. 536-544.

[16] D. M. Romero, B. Meeder, J. Kleinberg, Differences in the mechanics of information diffusion across topics: idioms, political hashtags, and complex contagion on twitter, in: Proceedings of the 20th international conference

[17] B. Stolz, H. Harrington, M. A. Porter, The topological 'shape' of brexit, arXiv preprint arXiv:1610.00752.

[18] P. N. Howard, B. Kollanyi, Bots,\# strongerin, and\# brexit: Computational propaganda during the uk-eu referendum, Available at SSRN 2798311.

${ }_{340}^{6}$ [19] M. Bianchetti, D. Galli, C. Ricci, A. Salvatori, M. Scaringi, Brexit or bremain? evidence from bubble analysis, Evidence from Bubble Analysis (June 20, 2016). 
[20] R. Steinberger, B. Pouliquen, E. Van Der Goot, An introduction to the europe media monitor family of applications, arXiv preprint arXiv:1309.5290.

[22] A. Clauset, M. E. Newman, C. Moore, Finding community structure in very large networks, Physical review E 70 (6) (2004) 066111.

[29] L. A. Adamic, N. Glance, The political blogosphere and the 2004 us election: divided they blog, in: Proceedings of the 3rd international workshop on Link discovery, ACM, 2005, pp. 36-43. 
[30] A. Bessi, F. Zollo, M. Del Vicario, M. Puliga, A. Scala, G. Caldarelli, detection algorithm [26, 27]. Results for the comparison are reported in Tab. 4 .

\begin{tabular}{|l|c|c|c|c|}
\hline & FG & WT & ML & LP \\
\hline \hline FG & 1 & 0.69 & 0.90 & 0.50 \\
WT & - & 1 & 0.63 & 0.32 \\
ML & - & - & 1 & 0.52 \\
LP & - & - & - & 1 \\
\hline
\end{tabular}

Table 4: The Rand similarity index among the partitions obtained by different community detection algorithms. 


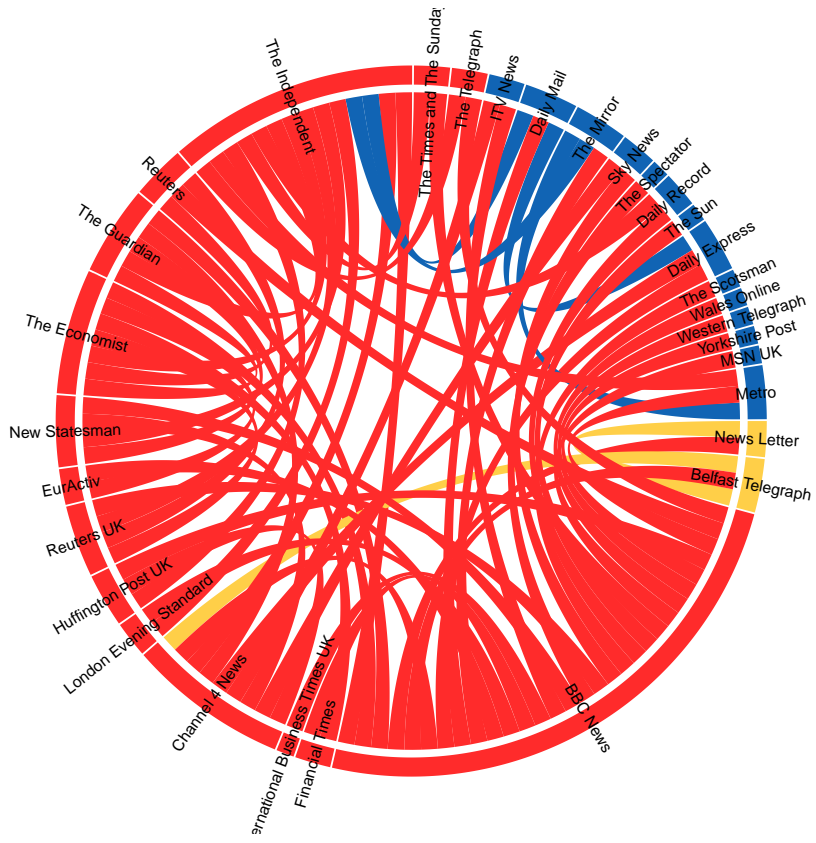

(a)

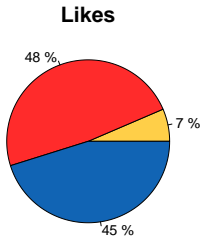

(b)

Figure 7: Backbone structure for the bipartite projection of the pages-users graph $G_{p}$ (a) and percentage of pages in the different communities (b). Colors indicate the membership of users in the different communities (blue for $\mathrm{C} 1$, red for $\mathrm{C} 2$, and yellow for $\mathrm{C} 3$ ) detected by the WT algorithm, while for the extraction of the backbone we considered the level of significance $\alpha=0.03$.

In Fig. 7(a) we show the backbone structure of $G_{p}$. Colors (resp., blue, red, and yellow) represent the membership to one of the three communities (resp., C1, C2, and C3) detected by the Walktrap (WT) algorithm (see Methods section for further details). Fig.7(b) reports the percentage of pages in each community. Differently from the FG algorithm, WT detects three main communities and five isolated nodes. However, the $27 \%$ of nodes belongs to the same community in both cases.

Our analysis underlines the spontaneous emergence of separate communities active on Brexit pages, where the connections among pages are a simple result of the interaction of users on them. 


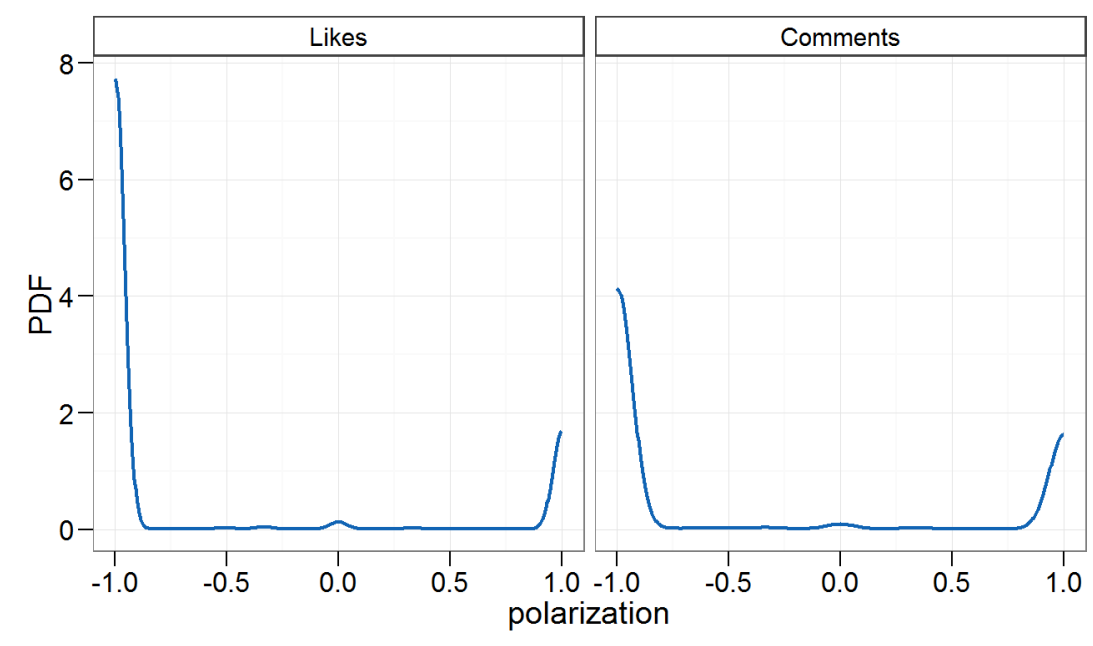

Figure 8: Probability density function (PDF) for the users polarization $\varrho(u)$ by likes (left) and by comments (right). $\varrho(u)=1$ (resp. $\varrho(u)=-1$ ) indicates that users $u$ is polarized towards C2 (resp., C1).

Taking into account the positive meaning of the like as a feedback to a post, we characterize how contents from the two biggest communities detected in $G_{p}$ are consumed by Facebook users. We define the users polarization by likes (reps., comments) as

$$
\varrho(u)=(y-x) /(y+x),
$$

where $y$ is the number of likes (resp., comments) that user $u$ left on posts of $\mathrm{C} 2$ and $x$ the number of likes (resp., comments) left on posts of C1. Thus, a user $u$ is said to be polarized towards C2 (resp., C1) if $\varrho(u)=1$ (resp., -1 ). In Fig. 8 we report the Probability Density Function (PDF) of users polarization by likes (left panel) and comments (right panel). We find that $\varrho(u)$ is sharply bimodal in both cases, denoting that the majority of users may be divided into two main groups referring to the two communities of Fig. 7(a).

Thus, we have shown that users form well segregated communities. We now want to compare their activities on posts from Brexit pages. In Fig. 9(a) we report the Complementary Cumulative Distribution Function (CCDF) of likes 
(left) and comments (right) made by users polarized towards both communities, while in Fig. 9(b) we report the CCDF of the lifetime of polarized users. The lifetime is defined as the temporal distance, in terms of days, between the first and last comment made by any given user. 


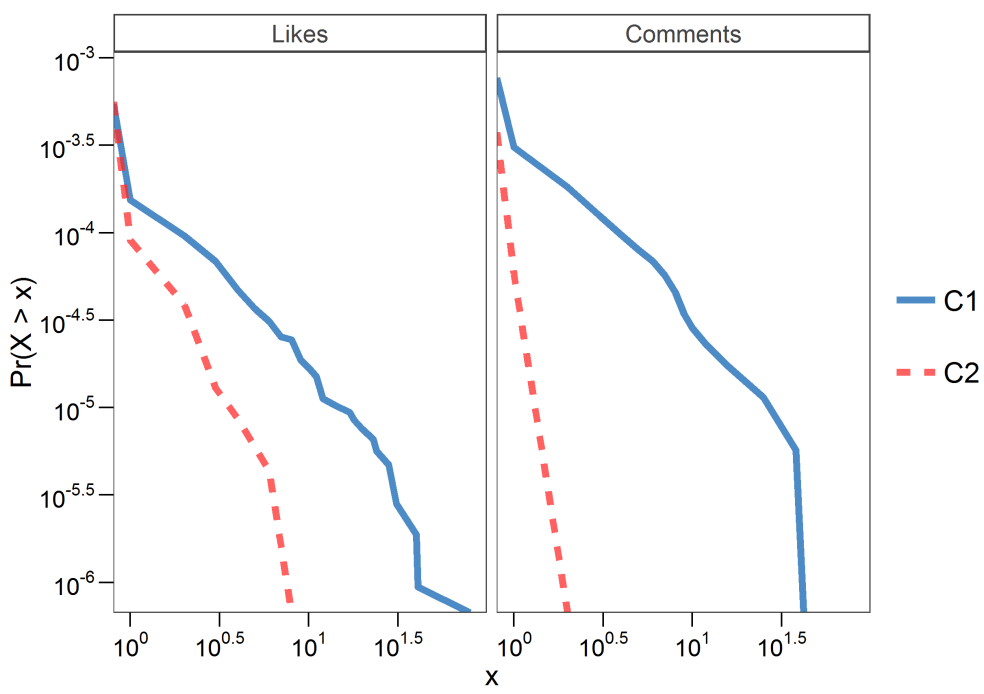

(a)

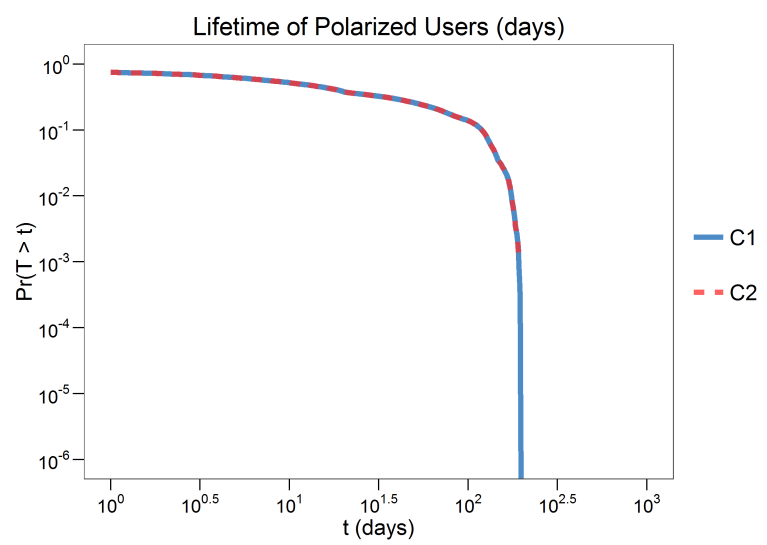

(b)

Figure 9: (a) CCDF of likes (left) and comments (right) made by users polarized towards C1 (solid blue) and towards $\mathrm{C} 2$ (dashed red). (b) CCDF of the lifetime of users polarized towards either $\mathrm{C} 1$ (solid blue) or 2 (dashed red). The lifetime is computed as the temporal distance, in terms of days, between the first and last comment made by any given user. 


\section{Appendix B}

In this section we provide the list of all Facebook pages of news sources whose legal head office (at least one of them) is located in the United Kingdom.

Pages engaged in the debate around Brexit are denoted by $\mathrm{Y}$ (N, otherwise), followed by the community to which they belong ( $\mathrm{C} 1$ or $\mathrm{C} 2)$.

\begin{tabular}{|l|l|r|c|}
\hline ID & Page Name & Facebook Code & Brexit \\
\hline \hline 1 & BBC News & 228735667216 & $\mathrm{Y}(\mathrm{C} 1)$ \\
2 & Channel 4 News & 6622931938 & $\mathrm{Y}(\mathrm{C} 1)$ \\
3 & Euractiv (English) & 15299247059 & $\mathrm{Y}(\mathrm{C} 1)$ \\
4 & Financial Times & 8860325749 & $\mathrm{Y}(\mathrm{C} 1)$ \\
5 & Huffingtonpost UK & 143753582359049 & $\mathrm{Y}(\mathrm{C} 1)$ \\
6 & International Business Times UK & 224377357631653 & $\mathrm{Y}(\mathrm{C} 1)$ \\
7 & New Economics Foundation & 110275553302 & $\mathrm{Y}(\mathrm{C} 1)$ \\
8 & New Statesman & 100959719644 & $\mathrm{Y}(\mathrm{C} 1)$ \\
9 & Open Europe Today & 321253057971308 & $\mathrm{Y}(\mathrm{C} 1)$ \\
10 & Reuters & 114050161948682 & $\mathrm{Y}(\mathrm{C} 1)$ \\
11 & Reuters UK & 208314602512037 & $\mathrm{Y}(\mathrm{C} 1)$ \\
12 & The Economist & 6013004059 & $\mathrm{Y}(\mathrm{C} 1)$ \\
13 & The Guardian UK & 10513336322 & $\mathrm{Y}(\mathrm{C} 1)$ \\
14 & The Independent & 13312631635 & $\mathrm{Y}(\mathrm{C} 1)$ \\
15 & The Register & 206419956048907 & $\mathrm{Y}(\mathrm{C} 1)$ \\
16 & WN.com & 229101503845879 & $\mathrm{Y}(\mathrm{C} 1)$ \\
17 & Belfast Telegraph & 237692023818 & $\mathrm{Y}(\mathrm{C} 2)$ \\
18 & Daily Express & 129617873765147 & $\mathrm{Y}(\mathrm{C} 2)$ \\
19 & Daily Mail & 164305410295882 & $\mathrm{Y}(\mathrm{C} 2)$ \\
20 & Daily Record & 187523381277554 & $\mathrm{Y}(\mathrm{C} 2)$ \\
21 & East Anglian Daily Times & 6478299951 & $\mathrm{Y}(\mathrm{C} 2)$ \\
22 & ITV News & 148007467671 & $\mathrm{Y}(\mathrm{C} 2)$ \\
23 & London Evening Standard & 358837740527 & $\mathrm{Y}(\mathrm{C} 2)$ \\
24 & Metro (UK) & MSN UK & $\mathrm{Y}(\mathrm{C} 2)$ \\
26 & News Letter & $\mathrm{Y}(\mathrm{C} 2)$
\end{tabular}




\begin{tabular}{|c|c|}
\hline 27 & Nottingham Post \\
\hline 28 & Sky News \\
\hline 29 & The Mirror \\
\hline 30 & The Scotsman \\
\hline 31 & The Spectator \\
\hline 32 & The Sun \\
\hline 33 & The Times and Sunday Times \\
\hline 34 & Wales Online \\
\hline 35 & Wandsworth Guardian \\
\hline 36 & Western Telegraph \\
\hline 37 & Yorkshire Post \\
\hline 38 & Airforce Technology Website \\
\hline 39 & Azo Mining \\
\hline 40 & BBC Radio \\
\hline 41 & Cafebabel (English) \\
\hline 42 & City A.M. \\
\hline 43 & Dunmow Broadcast \\
\hline 44 & EU business \\
\hline 45 & Euromoney \\
\hline 46 & European Railway Review \\
\hline 47 & Expatica \\
\hline 48 & Farming Life \\
\hline 49 & FCO - Foreign and Commonwealth Office \\
\hline 50 & Harborough Mail \\
\hline 51 & Herald Scotland \\
\hline 52 & Inmarsat \\
\hline 53 & Lydian International \\
\hline 54 & Mining Technology \\
\hline 55 & Mondo Visione \\
\hline 56 & MoneyWeek \\
\hline 57 & Monsters and Critics \\
\hline 58 & New Civil Engineer \\
\hline 59 & OneWorld.net: Palestine \\
\hline
\end{tabular}

\begin{tabular}{|c|c|}
\hline 309833935716287 & $\mathrm{Y}(\mathrm{C} 2)$ \\
\hline 164665060214766 & $\mathrm{Y}(\mathrm{C} 2)$ \\
\hline 6149699161 & $\mathrm{Y}(\mathrm{C} 2)$ \\
\hline 293226174987 & $\mathrm{Y}(\mathrm{C} 2)$ \\
\hline 111263798903232 & $\mathrm{Y}(\mathrm{C} 2)$ \\
\hline 161385360554578 & $\mathrm{Y}(\mathrm{C} 2)$ \\
\hline 147384458624178 & $\mathrm{Y}(\mathrm{C} 2)$ \\
\hline 21226447182 & $\mathrm{Y}(\mathrm{C} 2)$ \\
\hline 113349742029506 & $\mathrm{Y}(\mathrm{C} 2)$ \\
\hline 180521675319022 & $\mathrm{Y}(\mathrm{C} 2)$ \\
\hline 316795048375439 & $\mathrm{Y}(\mathrm{C} 2)$ \\
\hline 376588539031515 & $\mathrm{~N}$ \\
\hline 195005930530874 & $\mathrm{~N}$ \\
\hline 1470145583204820 & $\mathrm{~N}$ \\
\hline 357343795001 & $\mathrm{~N}$ \\
\hline 213682385348579 & $\mathrm{~N}$ \\
\hline 181182540669 & $\mathrm{~N}$ \\
\hline 215108901846669 & $\mathrm{~N}$ \\
\hline 192279900885723 & $\mathrm{~N}$ \\
\hline 404359882930504 & $\mathrm{~N}$ \\
\hline 206982432584 & $\mathrm{~N}$ \\
\hline 243070359106664 & $\mathrm{~N}$ \\
\hline 408582579294175 & $\mathrm{~N}$ \\
\hline 219817851378553 & $\mathrm{~N}$ \\
\hline 271154343382 & $\mathrm{~N}$ \\
\hline 317156988374684 & $\mathrm{~N}$ \\
\hline 186900121339682 & $\mathrm{~N}$ \\
\hline 326019370778750 & $\mathrm{~N}$ \\
\hline 169767016460715 & $\mathrm{~N}$ \\
\hline 110326662354766 & $\mathrm{~N}$ \\
\hline 193326863118 & $\mathrm{~N}$ \\
\hline 166793706822441 & $\mathrm{~N}$ \\
\hline 106968052697581 & $\mathrm{~N}$ \\
\hline
\end{tabular}




\begin{tabular}{|c|c|c|c|}
\hline 60 & Oxford Analytica & 160525917321265 & $\mathrm{~N}$ \\
\hline 61 & Pan European Networks & 230201663697109 & $\mathrm{~N}$ \\
\hline 62 & Publish What You Pay & 176624229034172 & $\mathrm{~N}$ \\
\hline 63 & Railway Magazine & 135345903226042 & $\mathrm{~N}$ \\
\hline 64 & Routes News & 126251777434574 & $\mathrm{~N}$ \\
\hline 65 & Seatrade Global & 470795739645931 & $\mathrm{~N}$ \\
\hline 66 & Survival International & 19668531552 & $\mathrm{~N}$ \\
\hline 67 & Tax-News & 375456009146619 & $\mathrm{~N}$ \\
\hline 68 & The Argus & 57197526698 & $\mathrm{~N}$ \\
\hline 69 & The Courier & 325681791214 & $\mathrm{~N}$ \\
\hline 70 & The International Institute for Strategic Studies & 29840385993 & $\mathrm{~N}$ \\
\hline 71 & The Scottish Government & 200786289976224 & $\mathrm{~N}$ \\
\hline 72 & The Telegraph & 143666524748 & $\mathrm{~N}$ \\
\hline 73 & The Visitor & 68554461041 & $\mathrm{~N}$ \\
\hline 74 & This is Africa & 779213412106756 & $\mathrm{~N}$ \\
\hline 75 & This is Derbyshire & 142370589115824 & $\mathrm{~N}$ \\
\hline 76 & This is Staffordshire & 11878899813 & $\mathrm{~N}$ \\
\hline 77 & Thomson Reuters Foundation & 31301735406 & $\mathrm{~N}$ \\
\hline 78 & World Fishing - The Magazine for Fishing & 552321618120006 & $\mathrm{~N}$ \\
\hline 79 & Cyprus Expat News & 357342727764507 & $\mathrm{~N}$ \\
\hline 80 & African Business Magazine & 114117578656259 & $\mathrm{~N}$ \\
\hline 81 & African Review & 507239115959583 & $\mathrm{~N}$ \\
\hline
\end{tabular}

Table 5: UK Facebook News Sources and Brexit Community

Membership: List of all Facebook pages of news sources whose legal head office (at least one of them) is located in the United Kingdom. Pages engaged in the debate around Brexit are denoted by $Y$, followed by the community to which they belong. 